\title{
A system of degenerate parabolic nonlinear PDE's: a new free boundary problem
}

\author{
MiCHIEL BERTSCH ${ }^{\dagger}$ \\ Istituto per le Applicazioni del Calcolo "Mauro Picone”, Consiglio Nazionale delle Ricerche, \\ Viale del Policlinico, 137, I-00161 Roma, Italy \\ Dipartimento di Matematica, Università degli Studi di Roma “Tor Vergata”, \\ Via della Ricerca Scientifica, I-00133 Roma, Italy \\ RoBERTA DAL PASSO ${ }^{\ddagger}$ AND CARLO NiTSCH ${ }^{\S}$ \\ Dipartimento di Matematica, Università degli Studi di Roma “Tor Vergata”, \\ Via della Ricerca Scientifica, I-00133 Roma, Italy
}

[Received 30 July 2004 and in revised form 8 April 2005]

\begin{abstract}
We prove existence of solutions of a new free boundary problem described by a system of degenerate parabolic equations. The problem arises in petroleum engineering and concerns fluid flows in diatomite rocks. The unknown functions represent the pressure of the fluid and a damage parameter of the porous rock. These quantities are not necessarily continuous on the free boundary, which considerably complicates the mathematical analysis.
\end{abstract}

Keywords: Nonlinear parabolic system; degenerate parabolic PDE's; nonlocal damage mechanics; oil engineering.

\section{Introduction}

In this paper we study the existence of compactly supported solutions of the system

$$
\begin{cases}\omega_{t}=\left(\omega^{\mu}(p-I)_{+}^{\beta} \omega_{x}\right)_{x}+A(1-\omega)(p-I)_{+}^{\gamma} & \text { in } \mathbb{R} \times \mathbb{R}^{+}, \\ p_{t}=\left(\omega^{\alpha} p_{x}\right)_{x} & \text { in } \mathbb{R} \times \mathbb{R}^{+},\end{cases}
$$

with initial data

$$
\omega(x, 0)=\omega_{0}(x), \quad p(x, 0)=p_{0}(x) \quad \text { in } \mathbb{R} .
$$

Here $\omega_{0}$ and $p_{0}$ are given nonnegative and bounded functions which vanish outside an interval $[a, b]$, and $\alpha, \beta, \gamma, \mu, A$ and $I$ are constants satisfying

$$
\alpha, \beta, \gamma>0, \quad \mu, A, I \geqslant 0 .
$$

Physically the problem is motivated by a mathematical model for fluid flow in porous rocks with very low permeability in the pristine state, such as diatomite rocks, introduced by Barenblatt,

\footnotetext{
${ }^{\dagger}$ E-mail: m.bertsch@iac.cnr.it

${ }^{\ddagger}$ E-mail: dalpasso@mat.uniroma2.it

${ }^{\S}$ Current address: Dipartimento di Matematica e Applicazioni "R. Caccioppoli”, Università degli Studi di Napoli "Federico II", Complesso Monte S. Angelo, Via Cintia, I-80126 Napoli, Italy. E-mail: carlo.nitsch@dma.unina.it
} 
Patzek, Prostokishin and Silin [3] and further developed in [2] and [12]. In [3] the authors stress the importance of the presence of microfractures caused by the fluid flow itself, and try to capture in their model the presence of interfaces, sharp boundaries between regions where $\omega$ vanishes (undamaged rocks) and regions where $\omega>0$ (damaged rocks). So $\omega$ is a damage parameter and $p$ is the total pressure of water and oil.

The constant $I$ is related to the strength of the rocks: if $p$ is below this value then no damage can accumulate or diffuse in the rocks. The exact value of $I$ can only be determined by experiments, and in principle we can only say that it is nonnegative, otherwise during the geologic time microcracks would have appeared in the rock. From the mathematical point of view $I=0$ and $I>0$ give different results (see also [5]).

In system (1) we have not taken into account the physical constraint of no damage healing, which is mathematically expressed by the condition $\omega_{t} \geqslant 0$. In the case of similar but simpler models concerning damage accumulation ([4, 11]) it has been shown that this constraint can be treated mathematically, but since the analysis of our system is already quite complicated we have preferred not to consider it in the present paper.

Roughly speaking, we can describe our main result as follows (for its precise statement we refer to Section 2). If $\omega_{0}>0$ and $p_{0}>0$ in $(a, b)$ and if, in the case $I>0, p>0$ intersects the level $I$ at most twice, then Problem (1)-(2) has a solution, $(\omega, p)$, which satisfies

$$
\begin{cases}\omega(x, t)>0 \text { and } p(x, t)>0 & \text { if } a(t)<x<b(t) \\ \omega(x, t)=p(x, t)=0 & \text { if } x<a(t), x>b(t) .\end{cases}
$$

Here $a(t)$ and $-b(t)$ are continuous nonincreasing functions, $a(0)=a$ and $b(0)=b$. In addition

$$
\omega(x, t)(p(x, t)-I)_{+} \rightarrow 0 \quad \text { as } x \searrow a(t) \text { and } x \nearrow b(t) \quad \text { for a.e. } t>0,
$$

and

$$
p>I \text { in }\{(x, t): a(t)<x<b(t)\} \quad \text { if } p_{0}>I \text { in }(a, b) .
$$

The latter result implies that, at least if $I>0, p$ may be discontinuous across the interfaces. In view of property (4) it is natural to ask whether both $\omega$ and $(p-I)_{+}$are continuous across the interfaces. An extensive analysis of traveling wave solutions of system (1) in [5] suggests that the answer heavily depends on the various parameters. In particular there exist traveling waves for which $\omega$ or $(p-I)_{+}$have jumps across the interfaces. We observe that if $I>0$ and $\omega$ is discontinuous across the interface, then the term $(p-I)_{+}^{\beta} \omega_{x}$ is not well defined across the interface, being the product of a discontinuous function and a measure. This indicates that the definition of a solution is a delicate issue. For general solutions of system (1)-(2) the problem how to analyze possible discontinuities at the free boundaries is completely open.

We list some other challenging open problems. We are not able to extend our existence result to the case of higher space dimensions:

$$
\left\{\begin{array}{l}
\omega_{t}=\operatorname{div}\left(\omega^{\mu}(p-I)_{+}^{\beta} \nabla \omega\right)+A(1-\omega)(p-I)_{+}^{\gamma}, \\
p_{t}=\operatorname{div}\left(\omega^{\alpha} \nabla p\right) ;
\end{array}\right.
$$

in particular the BV-estimates, which play an essential role in our analysis (see Section 3), are no longer available. Also the problem of the uniqueness of solutions is completely open, even in the one-dimensional case; this is hardly surprising, since even for much simpler systems of degenerate parabolic equations there is no systematic theory for the uniqueness of solutions. 
Both the list of open problems and the behavior of solutions near the free boundaries suggest that the degeneracies of the equations of system (1) make the problem much more difficult to analyze than "standard" degenerate parabolic equations such as the porous medium equation and the Stefan problem. For example, formally one could easily write the Rankine-Hugoniot conditions which determine the velocity of the free boundaries, but one has to distinguish several cases according to the occurrence of discontinuities. For the time being, there is no hope to give a rigorous proof of the free boundary conditions.

In [2], [3] and [12], it has been explained that the occurrence of a free boundary has been observed in huge oil fields in California (the observation is based on the phenomenon of subsidence). We emphasize that at this stage, validation of the model is impossible due to the lack of experimental data.

In Section 2 we collect the hypotheses on the data, the definition of a solution, and the main theorem. In Section 3 we approximate system (1) with a uniformly parabolic system and we use some a priori estimates to show the existence of a pair of limit functions $(\omega, p)$. In Section 4 we prove the basic support properties of $\omega$ and $p$, in particular the continuity of the functions $a(t)$ and $b(t)$. In Section 5 we prove that $(\omega, p)$ is a solution of our problem.

\section{The main results}

We shall often use concepts such as support, but since $\omega$ and $p$ are not always continuous it is useful to introduce the following notation.

\section{NOTATION.}

(i) If $f: \mathbb{R} \rightarrow[0, \infty)$ and $G=\{x \in \mathbb{R}: f(x)>0\}$, then

$$
\operatorname{supp} f:=\overline{\left\{x \in G: \lim _{\delta \rightarrow 0} \frac{1}{2 \delta} \mu(G \cap(x-\delta, x+\delta))>0\right\}} .
$$

Equivalently, the support can be defined as the complement of the largest open set where $f=0$ a.e.

(ii) If $g: D \subseteq \mathbb{R}^{n} \rightarrow[0, \infty)$, with $n \geqslant 1$, then

$$
\{g>0\}:=\operatorname{Int}\left\{x \in D: \liminf _{\rho \rightarrow 0} f_{B_{\rho}(x) \cap D} g>0\right\},
$$

where we have used the notation $f_{A} f:=|A|^{-1} \int_{A} f, B_{\rho}(x)$ being the ball of radius $\rho$ centered at $x$.

(iii) We set $Q_{T}:=\mathbb{R} \times(0, T]$ for all $T>0$.

Throughout the paper we assume that $\omega_{0}$ and $p_{0}$ satisfy the hypotheses

$$
\begin{gathered}
\left\{\begin{array}{l}
p_{0}, \omega_{0} \in \mathrm{BV}(\mathbb{R}), \\
p_{0} \geqslant 0, \quad 0 \leqslant \omega_{0} \leqslant 1 \quad \text { in } \mathbb{R},
\end{array}\right. \\
\begin{cases}\omega_{0}(x)>0 \text { and } p_{0}(x)>0 & \text { if } a<x<b, \\
\omega_{0}(x)=p_{0}(x)=0 & \text { if } x<a \text { or } x>b,\end{cases}
\end{gathered}
$$


and

$$
\left\{\begin{array}{l}
\text { there exists } \bar{\rho}>0 \text { such that for any } \rho \in[0, \bar{\rho}) \\
\text { the sets }\left\{x: \omega_{0}(x)>\rho\right\} \text { and }\left\{x: p_{0}(x)>I \pm \rho\right\} \\
\text { are intervals (if they are nonempty). }
\end{array}\right.
$$

Before stating the main result we have to specify what we mean by solution. As we explained in the introduction, it is not obvious how to define in $\mathbb{R} \times \mathbb{R}^{+}$the products involving $\omega_{x}$ and $p_{x}$. Therefore we restrict the domains of integration in some of the integrals appearing in the definition of solution.

Definition 2.1 A pair $(\omega, p)$ that belongs to $X_{T}^{2}:=\left[L^{\infty}((0, T) ; \mathrm{BV}(\mathbb{R})) \cap L^{\infty}([0, T] \times \mathbb{R}) \cap\right.$ $\left.H^{1}\left((0, T) ; H^{-1}(\mathbb{R})\right)\right]^{2}$ for all $T>0$ is a solution of system (1)-2) if

(i) $\omega_{x} \in L_{\text {loc }}^{2}(\{p>I\}), p_{x} \in L_{\text {loc }}^{2}(\{\omega>0\})$,

(ii) $\omega^{\alpha} p_{x}^{2} \in L^{1}(\{\omega>0\})$ and $\omega^{\mu}(p-I)^{\beta} \omega_{x}^{2} \in L^{1}(\{p>I\})$,

(iii) for all $T>0$ and $\phi, \psi \in H^{1}(\mathbb{R} \times[0, T])$ with compact support (i.e. for some $M>0$, $\phi=\psi=0$ for a.e. $|x|>M)$ and for all $t \in[0, T]$,

$$
\int_{\mathbb{R}} \phi(t) p(t)-\int_{\mathbb{R}} \phi(0) p_{0}=\int_{0}^{t} \int_{\mathbb{R}} \phi_{t} p-\iint_{\{\omega>0\} \cap Q_{t}} \phi_{x} \omega^{\alpha} p_{x}
$$

and

$$
\begin{aligned}
\int_{\mathbb{R}} \psi(t) \omega(t) & -\int_{\mathbb{R}} \psi(0) \omega_{0} \\
& =\int_{0}^{t} \int_{\mathbb{R}} \psi_{t} \omega-\iint_{\{p>I\} \cap Q_{t}} \psi_{x} \omega^{\mu}(p-I)^{\beta} \omega_{x}+A \int_{0}^{t} \int_{\mathbb{R}} \psi(p-I)_{+}^{\gamma}(1-\omega) .
\end{aligned}
$$

Remark 2.2 Since $X_{T}^{2}$ is embedded in $\left[C\left([0, T] ; L_{\text {loc }}^{2}(\mathbb{R}) \text { ) }\right]^{2}\right.$ (see proof of Proposition 3.1 for more details), the integral equalities in (iii) are well defined for all $t \in[0, T]$.

Now we are ready to state the main result.

Theorem 2.3 Let hypotheses (3), (9), (10) and (11) be satisfied. Then Problem (1)-22) has a solution $(\omega, p)$ which has the following properties:

(i) there exist $a, b \in C([0, \infty))$ such that $a(0)=a, b(0)=b, a(t)$ is nonincreasing, $b(t)$ is nondecreasing, and

$$
\begin{array}{ll}
\omega(x, t)>0 \text { and } p(x, t)>0 & \text { if } a(t)<x<b(t), \\
\omega(x, t)=p(x, t)=0 & \text { if } x<a(t) \text { or } x>b(t) ;
\end{array}
$$

(ii) for a.e. $t>0, \omega(p-I)_{+} \rightarrow 0$ as $x \searrow a(t)$ and $x \nearrow b(t)$;

(iii) if $p_{0} \geqslant I$ in $(a, b)$ then $p(x, t) \geqslant I$ if $a(t)<x<b(t)$.

\section{A nondegenerate system}

We introduce, for $\varepsilon>0$, the problem

$$
\begin{cases}p_{t}=\left(\left(\omega^{\alpha}+\varepsilon\right) p_{x}\right)_{x} & \text { in } \mathbb{R} \times \mathbb{R}^{+} \\ \omega_{t}=\left(\left(\omega^{\mu}(p-I)_{+}^{\beta}+\varepsilon\right) \omega_{x}\right)_{x}+A(1-\omega)(p-I)_{+}^{\gamma} & \text { in } \mathbb{R} \times \mathbb{R}^{+} \\ p(x, 0)=p_{0}(x), \quad \omega(x, 0)=\omega_{0}(x) & \text { for } x \in \mathbb{R} .\end{cases}
$$


A weak solution of $(12)$ is defined in a standard way (requiring that $0 \leqslant \omega_{0} \leqslant 1, p_{0} \geqslant 0$ and $\left.\omega_{x}, p_{x} \in L_{\mathrm{loc}}^{2}(\mathbb{R} \times[0, \infty))\right)$.

PROPOSITION 3.1 Let $\omega_{0}$ and $p_{0}$ satisfy

$$
\left\{\begin{array}{l}
0 \leqslant \omega_{0} \leqslant 1, p_{0} \geqslant 0 \quad \text { in } \mathbb{R}, \\
\omega_{0}, p_{0} \in \mathrm{BV}(\mathbb{R}) \cap L^{q}(\mathbb{R}) \quad(1 \leqslant q \leqslant \infty) .
\end{array}\right.
$$

Then, for all $\varepsilon>0$, problem (12) has a weak solution $\left(\omega_{\varepsilon}, p_{\varepsilon}\right)$ satisfying

(i) $0 \leqslant \omega_{\varepsilon} \leqslant 1$ and $0 \leqslant p_{\varepsilon} \leqslant C$ in $\mathbb{R} \times[0, \infty)$;

(ii) for all $T>0$ there exists $C_{T}>0$ such that

$$
\int_{0}^{T} \int_{\mathbb{R}}\left(\omega_{\varepsilon}^{\mu}\left(p_{\varepsilon}-I\right)^{\beta}+\varepsilon\right) \omega_{\varepsilon x}^{2} \mathrm{~d} x \mathrm{~d} t+\int_{0}^{T} \int_{\mathbb{R}}\left(\omega_{\varepsilon}^{\alpha}+\varepsilon\right) p_{\varepsilon x}^{2} \mathrm{~d} x \mathrm{~d} t \leqslant C_{T} ;
$$

(iii) $\omega_{\varepsilon}$ and $p_{\varepsilon}$ are uniformly bounded in

$$
L^{\infty}\left((0, T) ; L^{q}(\mathbb{R})\right) \cap H^{1}\left((0, T) ; H^{-1}(\mathbb{R})\right) \cap L^{\infty}((0, T) ; \mathrm{BV}(\mathbb{R}))
$$

for all $1 \leqslant q \leqslant \infty$ and $T>0$;

(iv) for all $T>0$,

$$
\left(\omega_{\varepsilon}\left(p_{\varepsilon}-I\right)_{+}\right)^{v}
$$

is uniformly bounded in $L^{2}\left((0, T) ; H^{1}(\mathbb{R})\right)$ if $v \geqslant \frac{1}{2} \min \{\alpha+1, \beta+1, \mu+2\}$.

Proof. The proof consists of two steps.

Step 1. We assume that problem (12) has a smooth solution $\left(\omega_{\varepsilon}, p_{\varepsilon}\right)$. Then (i) follows from the comparison principle. Multiplying the equation for $p_{\varepsilon}$ by $p_{\varepsilon}^{q-1}(1 \leqslant q<\infty)$ we obtain a uniform bound of $p_{\varepsilon}$ in $L^{\infty}\left((0, \infty) ; L^{q}(\mathbb{R})\right)$. Multiplying the equation for $\omega_{\varepsilon}$ by $\omega_{\varepsilon}^{q-1}$ and using the bound for $p_{\varepsilon}$, we find that $\omega_{\varepsilon}$ is uniformly bounded in $L^{\infty}\left((0, T) ; L^{q}(\mathbb{R})\right)$. In particular, in the case $q=2$, this procedure yields (14).

Since $\left((p-I)_{+}\right)_{x}^{2} \leqslant p_{\varepsilon x}^{2}$, it follows from 14 that $\omega_{\varepsilon}^{\alpha}\left((p-I)_{+}\right)_{x}^{2}$ and $\omega_{\varepsilon}^{\mu}(p-I)^{\beta} \omega_{\varepsilon x}^{2}$ are uniformly bounded in $L^{1}(\mathbb{R} \times[0, T])$. By Hölder's and Young's inequalities this implies at once that

$$
\left(\left(\omega_{\varepsilon}\left(p_{\varepsilon}-I\right)_{+}\right)^{v}\right)_{x}
$$

is uniformly bounded in $L^{2}(\mathbb{R} \times[0, T])$ if $2 v \geqslant \alpha+1,2 v \geqslant \beta+1$ and $2 v \geqslant \mu+2$, and we have proved (iv). It follows from (14) and the equations for $\omega_{\varepsilon}$ and $p_{\varepsilon}$ that $\omega_{\varepsilon t}$ and $p_{\varepsilon t}$ are uniformly bounded in $L^{2}\left((0, T) ; H^{-1}(\mathbb{R})\right)$.

Finally, we prove the uniform boundedness in $L^{\infty}((0, T) ; \mathrm{BV}(\mathbb{R}))$. In order to estimate $p_{\varepsilon x}$ and $\omega_{\varepsilon x}$ we adopt a standard technique and introduce a smooth function $\eta_{\delta}$ whose derivative is an approximation of the sign function:

$$
\eta_{\delta}^{\prime}(s)= \begin{cases}-1 & \text { if } s<-\delta \\ 1 & \text { if } s>\delta\end{cases}
$$

We assume that $\eta_{\delta}$ is even, $\eta_{\delta}^{\prime \prime} \geqslant 0$ and $\eta_{\delta} \in C^{\infty}$. Multiplying the equation for $p_{\varepsilon x}$ by $\eta_{\delta}^{\prime}\left(p_{\varepsilon x}\right)$ and integrating yields

$$
\frac{\mathrm{d}}{\mathrm{d} t} \int_{\mathbb{R}} \eta_{\delta}\left(p_{\varepsilon x}\right) \mathrm{d} x=\int_{\mathbb{R}} \eta_{\delta}^{\prime}\left(p_{\varepsilon x}\right)\left(\left(\omega_{\varepsilon}^{\alpha}+\varepsilon\right) p_{\varepsilon x}\right)_{x x} \mathrm{~d} x \leqslant-\int_{\mathbb{R}} \eta_{\delta}^{\prime \prime}\left(p_{\varepsilon x}\right) p_{\varepsilon x x}\left(\omega_{\varepsilon}^{\alpha}\right)_{x} p_{\varepsilon x} \mathrm{~d} x .
$$


For fixed $\varepsilon>0$, the latter term vanishes as $\delta \rightarrow 0$ and we see that $\int_{\mathbb{R}}\left|p_{\varepsilon x}\right|$ is nonincreasing in time and hence equibounded. The proof for $\omega_{\varepsilon}$ is similar, and it is enough to observe that the term $A \eta_{\delta}^{\prime}\left(\omega_{\varepsilon x}\right)\left(1-\omega_{\varepsilon}\right)\left(1-p_{\varepsilon}\right)_{+}^{\gamma}$ is uniformly bounded in $L^{\infty}\left((0, T) ; L^{1}(\mathbb{R})\right)$.

Step 2. For fixed $\varepsilon>0$ we approximate the nonlinearities $\omega^{\alpha}, \omega^{\mu}(p-I)_{+}^{\beta}$ and $(p-I)_{+}^{\gamma}$ by smooth functions of $\omega$ and $p$, and the initial functions $\omega_{0}$ and $p_{0}$ by smooth functions of $x$. This leads to a family of problems, say problems $P_{\varepsilon, k}$, and following the proof in Step 1 we obtain a priori estimates for problem $P_{\varepsilon, k}$ which are similar to (i)-(ii)-(iii).

From Theorem 1 of [1], it follows that problem $P_{\varepsilon, k}$ admits a unique maximal classical solution in some interval $\left(0, \tau_{\varepsilon, k}\right)$, which can be continued as long as it remains bounded ([1, Theorem 3]). Hence it exists for all $t>0$. Since for every compact subset $U \subset \mathbb{R}$,

$$
\mathrm{BV}(\mathbb{R}) \cap L^{2}(\mathbb{R}) \hookrightarrow \hookrightarrow L^{2}(U) \hookrightarrow\left(W^{1,2}(U)\right)^{\prime}
$$

it follows from (ii) and Corollary 4 in [13] that the corresponding solutions $\left\{\left(\omega_{\varepsilon, k}, p_{\varepsilon, k}\right)\right\}$ are relatively compact in $\left[C\left([0, T] ; L_{\text {loc }}^{2}(\mathbb{R})\right)\right]^{2}$. Since the spatial derivatives are uniformly bounded in $L^{2}(\mathbb{R} \times[0, T])$, convergence along a subsequence yields a weak solution, $\left\{\left(\omega_{\varepsilon}, p_{\varepsilon}\right)\right\}$, of 12 . This completes the proof of Proposition 3.1

The compactness argument in $\left[C\left([0, T] ; L_{\text {loc }}^{2}(\mathbb{R})\right]^{2}\right.$ which we have used before yields a limit function $(\omega, p)$ which is a candidate for a solution of (1)-2):

TheOREM 3.2 Let $\omega_{0}$ and $p_{0}$ satisfy hypothesis $9-10$ and let $\left(\omega_{\varepsilon}, p_{\varepsilon}\right)$ be defined by Proposition 3.1 Then there exist a sequence $\varepsilon_{n} \rightarrow 0$ and functions $\omega$ and $p$ such that for all $T>0$,

$$
\left(\omega_{\varepsilon_{n}}, p_{\varepsilon_{n}}\right) \rightarrow(\omega, p) \quad \text { in }\left[C\left([0, T] ; L_{\text {loc }}^{2}(\mathbb{R})\right)\right]^{2},
$$

and

(i) $0 \leqslant \omega \leqslant 1$ and $0 \leqslant p \leqslant C$ in $\mathbb{R} \times[0, T]$;

(ii) $\omega, p \in C\left([0, T] ; L^{q}(\mathbb{R})\right) \cap H^{1}\left((0, T) ; H^{-1}(\mathbb{R})\right) \cap L^{\infty}((0, T) ; \mathrm{BV}(\mathbb{R})), 1 \leqslant q<\infty$.

\section{Support properties}

In this section we prove that the functions $\omega$ and $p$ defined by Theorem 3.2 satisfy

$$
\operatorname{supp} \omega(t)=\operatorname{supp} p(t)=[a(t), b(t)] \quad \text { if } t \geqslant 0,
$$

where

$$
a, b \in C([0, \infty)), \quad a(0)=a, \quad b(0)=b,
$$

and

$$
a \text { is nonincreasing and } b \text { nondecreasing in }[0, \infty) \text {. }
$$

In addition

$$
\text { if } b\left(t_{2}\right)>b\left(t_{1}\right) \text { for some } 0 \leqslant t_{1} \leqslant t_{2} \text {, then }\{p>I\} \cap\left(\left(b\left(t_{1}\right), b\left(t_{2}\right)\right) \times\left(t_{1}, t_{2}\right)\right) \neq \emptyset .
$$

We remind the reader the notation (7) for the support.

The proof of these results consists of several lemmas. 
Lemma 4.1 If $\bar{x} \notin \operatorname{supp} \omega(\bar{t})$ for some $(\bar{x}, \bar{t}) \in \mathbb{R} \times \mathbb{R}^{+}$, then there exists a neighborhood $U(\bar{x})$ of $\bar{x}$ such that $\omega=0$ a.e. in $U(\bar{x}) \times[0, \bar{t}]$. The same result holds if we replace $\omega$ by $p$.

Proof. Since supp $\omega(\bar{t})$ is closed, there exists a neighborhood $U(\bar{x})$ of $\bar{x}$ such that $U(\bar{x}) \cap$ $\operatorname{supp} \omega(\bar{t})=\emptyset$. Let $\left(\omega_{\varepsilon}, p_{\varepsilon}\right)$ be the solution of the nondegenerate problem $(12)$. We multiply the equation for $\omega_{\varepsilon}$ by $-\varphi^{2}(x) /\left(\omega_{\varepsilon}+\delta\right)$, where $\delta>0$ and $\varphi$ is a Lipschitz continuous function such that $\operatorname{supp} \varphi \subseteq U(\bar{x})$. Integrating by parts shows that for $0 \leqslant t \leqslant \bar{t}$,

$$
\begin{aligned}
&-\left.\int_{\mathbb{R}} \varphi^{2} \log \left(\omega_{\varepsilon}+\delta\right)\right|_{t} ^{\bar{t}}+A \int_{t}^{\bar{t}} \int_{\mathbb{R}} \varphi^{2} \frac{\left(1-\omega_{\varepsilon}\right)\left(p_{\varepsilon}-I\right)_{+}^{\gamma}}{\omega_{\varepsilon}+\delta} \\
&=-2 \int_{t}^{\bar{t}} \int_{\mathbb{R}} \varphi \varphi_{x}\left(\omega^{\mu}\left(p_{\varepsilon}-I\right)_{+}^{\beta}+\varepsilon\right)\left(-\log \left(\omega_{\varepsilon}+\delta\right)\right)_{x} \\
&-\int_{t}^{\bar{t}} \int_{\mathbb{R}} \varphi^{2}\left[\left(-\log \left(\omega_{\varepsilon}+\delta\right)\right)_{x}\right]^{2}\left(\omega_{\varepsilon}^{\mu}\left(p_{\varepsilon}-I\right)_{+}^{\beta}+\varepsilon\right) \\
& \leqslant \int_{t}^{\bar{t}} \int_{\mathbb{R}} \varphi_{x}^{2}\left(\omega_{\varepsilon}^{\mu}\left(p_{\varepsilon}-I\right)_{+}^{\beta}+\varepsilon\right) \leqslant C(\varphi)(t-\bar{t}),
\end{aligned}
$$

where we have used Hölder's and Young's inequalities. By 15 we have, for fixed $\delta>0$,

$$
\begin{aligned}
-\int_{\mathbb{R}} \varphi^{2} \log (\omega+\delta)(\bar{t}) & +A \int_{t}^{\bar{t}} \int_{\mathbb{R}} \varphi^{2} \frac{(1-\omega)(p-I)_{+}^{\gamma}}{\omega+\delta} \\
& \leqslant-\int_{\mathbb{R}} \varphi^{2} \log (\omega+\delta)(t)+C(\varphi)(\bar{t}-t)
\end{aligned}
$$

and since $\delta$ is arbitrary $\omega$ and $p$ satisfy

$$
-\int_{\mathbb{R}} \varphi^{2} \log \omega(\bar{t})+A \int_{t}^{\bar{t}} \int_{\mathbb{R}} \varphi^{2} \frac{(1-\omega)(p-I)_{+}^{\gamma}}{\omega} \leqslant-\int_{\mathbb{R}} \varphi^{2} \log \omega(t)+C(\varphi)(\bar{t}-t) .
$$

Since $\omega(\bar{t})=0$ in $\operatorname{supp} \varphi$ this implies that

$$
-\int_{\mathbb{R}} \varphi(x)^{2} \log \omega(x, t)=+\infty \quad \text { for } 0 \leqslant t<\bar{t} .
$$

Hence the set $\{x \in U(\bar{x}): \omega(x, t)=0\}$ is dense in $U(\bar{x})$, and since $\omega \in L^{\infty}((0, \bar{t}) ; \mathrm{BV}(\mathbb{R}))$ this means that $\omega(x, t)=0$ for all $0 \leqslant t<\bar{t}$ and for a.e. $x \in U(\bar{x})$. To complete the proof of Lemma 4.1 it is enough to repeat the same argument for $p$.

LEMMA 4.2 For all $t>0$,

$$
\operatorname{supp} \omega(t)=\operatorname{supp} p(t)
$$

In addition

$$
\operatorname{supp} \omega\left(t_{1}\right) \subseteq \operatorname{supp} \omega\left(t_{2}\right) \quad \text { if } 0 \leqslant t_{1}<t_{2} .
$$

Proof. Property 23] follows at once from Lemma 4.1. We claim that

$$
\operatorname{supp} p(t) \subseteq \operatorname{supp} \omega(t) \quad \text { for } t \geqslant 0 .
$$


Taking $(\bar{x}, \bar{t})$ and $U(\bar{x})$ as in Lemma 4.1 , it is enough to prove that

$$
p(x, t)=0 \quad \text { for a.e. } x \in U(\bar{x}), 0 \leqslant t \leqslant \bar{t} .
$$

Since, by Lemma 4.1, $\omega(x, t)=0$ for a.e. $x \in U(\bar{x})$ if $0 \leqslant t \leqslant \bar{t}$, it follows easily from the equation for $p$ that

$$
p(x, t)=p_{0}(x) \quad \text { for a.e. } x \in U(\bar{x}), 0 \leqslant t \leqslant \bar{t} .
$$

Indeed, for any smooth and nonnegative function $\psi$ in $\mathbb{R}$ such that supp $\psi \subseteq U(\bar{x})$, we see, by (14) and Hölder's inequality, that for all $0<t \leqslant \bar{t}$,

$$
\begin{aligned}
\left|\int_{\mathbb{R}} p_{\varepsilon}(t) \psi-\int_{\mathbb{R}} p_{0 \varepsilon} \psi\right| & =\left|\int_{0}^{t} \int_{\mathbb{R}} \psi_{x}\left(\omega_{\varepsilon}^{\alpha}+\varepsilon\right) p_{\varepsilon x}\right| \\
& \leqslant C\left(\int_{0}^{t} \int_{\mathbb{R}} \psi_{x}^{2}\left(\omega_{\varepsilon}^{\alpha}+\varepsilon\right)\right)^{1 / 2} \rightarrow 0 \quad \text { as } \varepsilon \rightarrow 0,
\end{aligned}
$$

and 25) follows. Since $U(\bar{x}) \cap \operatorname{supp} p_{0}=U(\bar{x}) \cap \operatorname{supp} \omega_{0}=\emptyset$, it follows from (24) that $p(x, t)=0$ for a.e. $x \in U(\bar{x})$ if $0 \leqslant t \leqslant \bar{t}$. This proves 24 .

Exchanging the roles of $\omega$ and $p$ we find that $\operatorname{supp} \omega(t) \subseteq \operatorname{supp} p(t)$, and the proof is complete.

LEMMA 4.3 For all $t>0$ the set $\operatorname{supp} \omega(t)$ is connected.

Proof. Arguing by contradiction we assume that there exist $x_{0} \in \mathbb{R}$ and $t_{0}>0$ such that

$x_{0} \notin \operatorname{supp} \omega\left(t_{0}\right), \quad\left(-\infty, x_{0}\right) \cap \operatorname{supp} \omega\left(t_{0}\right) \neq \emptyset, \quad\left(x_{0}, \infty\right) \cap \operatorname{supp} \omega\left(t_{0}\right) \neq \emptyset$.

By [24), $x_{0} \notin \operatorname{supp} \omega_{0}=[a, b]$. Without loss of generality we may assume that $x_{0}>b$. Since the supports of $\omega$ and $p$ coincide, we obtain a contradiction if we prove that

$$
p\left(x, t_{0}\right)=0 \quad \text { for a.e. } x>x_{0} .
$$

In view of Lemmas 4.1 and 4.2 there exists a neighborhood $\left(x_{0}-\delta, x_{0}+\delta\right)$ of $x_{0}$ such that

$$
\omega=p=0 \quad \text { a.e. in }\left(x_{0}-\delta, x_{0}+\delta\right) \times\left(0, t_{0}\right) .
$$

Let $\phi \in C^{\infty}(\mathbb{R})$ be a nonnegative function such that

$$
\phi=0 \quad \text { if } x<x_{0}-\delta \text { and } \phi(x)=1 \quad \text { if } x \geqslant x_{0} .
$$

Then

$$
\begin{aligned}
\int_{x_{0}}^{\infty} p_{\varepsilon}\left(x, t_{0}\right) & \leqslant \int_{x_{0}-\delta}^{\infty} \phi(x) p_{\varepsilon}\left(x, t_{0}\right) \\
& =\int_{x_{0}-\delta}^{\infty} \phi(x) p_{0 \varepsilon}(x)-\int_{0}^{t_{0}} \int_{x_{0}-\delta}^{x_{0}} \phi^{\prime}\left(\omega_{\varepsilon}^{\alpha}+\varepsilon\right) p_{\varepsilon x} .
\end{aligned}
$$

Since $p_{0}=0$ in $\left(x_{0}-\delta, \infty\right)$, the first term on the right hand side vanishes as $\delta \rightarrow 0$. The second term is bounded by

$$
\left(\int_{0}^{t} \int_{x_{0}-\delta}^{x_{0}}\left(\omega_{\varepsilon}^{\alpha}+\varepsilon\right) p_{\varepsilon x}^{2}\right)^{1 / 2}\left(\int_{0}^{t} \int_{x_{0}-\delta}^{x_{0}}\left(\omega_{\varepsilon}^{\alpha}+\varepsilon\right)\left(\phi^{\prime}(x)\right)^{2}\right)^{1 / 2},
$$

which, in view of (14) and 27), vanishes as $\varepsilon \rightarrow 0$. Hence 26 follows from 28.

Combined with the following theorem, Lemmas 4.14 .3 imply (17)-(18). 
TheOREM 4.4 Let $(\omega, p)$ be a weak solution of Problem (1)-(2) constructed in Theorem 2.3 . There exists a nondecreasing function $r \in C([0, \infty) ;[0, \infty))$ with $r(0)=0$ such that if supp $\omega_{0} \subseteq$ $B\left(x_{0}, r_{0}\right)$ then $\operatorname{supp} \omega(t) \subseteq B\left(x_{0}, r_{0}+r(t)\right)$.

In other words, Theorem 4.4 states the property of finite speed of propagation for solutions of Problem (1)-(2). The proof of this theorem is postponed to the Appendix.

Finally, we prove property [19]. Arguing by contradiction, we assume

$$
\{p>I\} \cap\left(\left(b\left(t_{1}\right), b\left(t_{2}\right)\right) \times\left(t_{1}, t_{2}\right)\right)=\emptyset .
$$

Testing the equation for $\omega_{\varepsilon}$ with a smooth, nonnegative function $\varphi:\left(b\left(t_{1}\right), b\left(t_{2}\right)\right) \rightarrow \mathbb{R}$ with compact support yields

$$
\begin{aligned}
\int_{\mathbb{R}} \varphi \omega_{\varepsilon}(t)-\int_{\mathbb{R}} \varphi \omega_{\varepsilon}\left(t_{1}\right) & =-\int_{t_{1}}^{t} \int_{\mathbb{R}} \varphi_{x}\left(\omega_{\varepsilon}^{\mu}\left(p_{\varepsilon}-I\right)_{+}^{\beta}+\varepsilon\right) \omega_{\varepsilon x}+A \int_{t_{1}}^{t} \int_{\mathbb{R}} \varphi\left(p_{\varepsilon}-I\right)_{+}^{\gamma}\left(1-\omega_{\varepsilon}\right) \\
& \leqslant C\left(\int_{t_{1}}^{t_{2}} \int_{b\left(t_{1}\right)}^{b\left(t_{2}\right)}\left(\omega_{\varepsilon}^{\mu}\left(p_{\varepsilon}-I\right)_{+}^{\beta}+\varepsilon\right)^{1 / 2}+\int_{t_{1}}^{t_{2}} \int_{b\left(t_{1}\right)}^{b\left(t_{2}\right)}\left(p_{\varepsilon}-I\right)_{+}^{\gamma}\right)
\end{aligned}
$$

for all $t_{1}<t \leqslant t_{2}$. Here we have used (14). If we let $\varepsilon \rightarrow 0$, the right hand side vanishes, and we obtain

$$
\int_{\mathbb{R}} \varphi \omega_{\varepsilon}(t)=\int_{\mathbb{R}} \varphi \omega_{\varepsilon}\left(t_{1}\right)=0 \quad \text { for all } t \in\left(t_{1}, t_{2}\right] .
$$

Since $\varphi$ is arbitrary, we get

$$
\int_{b\left(t_{1}\right)}^{b\left(t_{2}\right)} \omega_{\varepsilon}(t)=0 \quad \text { for all } t \in\left(t_{1}, t_{2}\right],
$$

which implies $b\left(t_{1}\right)=b\left(t_{2}\right)$, and we have found a contradiction.

\section{Proof of the main result}

In this section we show that the pair $(\omega, p)$, defined by Theorem 3.2, is a solution of problem (1)-(2) and has all properties listed in Theorem 2.3 We shall need condition (11) on the initial functions, and without loss of generality we may assume that for all $\varepsilon>0$,

$$
\omega_{0 \varepsilon} \text { and } p_{0 \varepsilon} \quad \text { satisfy condition }(11) \text {. }
$$

It follows from standard lap-number theory ([10]) that for all $t>0$ and $0 \leqslant \rho<\bar{\rho}$,

$$
\left\{x: p_{\varepsilon}(x, t)>I \pm \rho\right\} \quad \text { is an interval, }
$$

and hence, by (15), we find that $\{x: p(t)>I \pm \rho\}$ is an interval.

This section is organized as follows. First we prove Theorem 2.3 except property (iii), assuming that the following result holds:

Proposition 5.1 Let $(\omega, p)$ and the function $a(t)$ and $b(t)$ be defined by, respectively, Theorem 3.2 and $16-18$. Then for any compact set

$$
K \subset\{(x, t): a(t)<x<b(t), t \geqslant 0\}
$$

there exists $\delta_{K}>0$ such that

$$
\omega_{\varepsilon} \geqslant \delta_{K} \quad \text { in } K \text { for all } 0 \leqslant \varepsilon \leqslant \varepsilon_{K} .
$$

In particular it follows that $\{\omega>0\}=\{(x, t): a(t)<x<b(t), t \geqslant 0\}$. 
Proof of Theorem 2.3. In view of Theorem 3.2 $(\omega, p)$ is a solution of problem (1)-(2) if we show that it has properties (ii) and (iii) of Definition 2.1. By (31) and standard results on bounded weak solutions of uniformly parabolic equations ([9]), the functions $\left\{p_{\varepsilon}, \varepsilon>0\right\}$ are uniformly Hölder continuous in any compact set $K \subseteq\{\omega>0\} \cap(\mathbb{R} \times(0, T))$. Hence, the functions $\left\{\omega_{\varepsilon}, \varepsilon>0\right\}$ are uniformly Hölder continuous in compact subsets of $\{p>I\} \cap(\mathbb{R} \times(0, T))$ (here we have used the fact that the supports of $\omega$ and $p$ coincide). In particular

$$
\omega_{\varepsilon x} \rightarrow \omega_{x} \quad \text { weakly in } L_{\text {loc }}^{2}(\{p>I\}), \quad p_{\varepsilon x} \rightarrow p_{x} \quad \text { weakly in } L_{\text {loc }}^{2}(\{\omega>0\}) .
$$

Combining (14, , 15) and (32) we obtain, for all $T>0$,

$$
\iint_{\{p>I\} \cap Q_{T}} \omega^{\mu}(p-I)^{\beta} \omega_{x}^{2}+\iint_{\{\omega>0\} \cap Q_{T}} \omega^{\alpha} p_{x}^{2}<C_{T},
$$

where the constant $C_{T}$ depends only on $T$, which implies (ii) of Definition 2.1

To prove (iii), we observe that for every $\varphi$ smooth enough, with compact support in $\mathbb{R} \times[0, T]$,

$$
\int_{\mathbb{R}} \varphi(t) p_{\varepsilon}(t)-\int_{\mathbb{R}} \varphi(0) p_{0}-\int_{0}^{t} \int_{\mathbb{R}} \varphi_{t} p_{\varepsilon}=-\int_{0}^{t} \int_{\mathbb{R}} \varphi_{x}\left(\omega_{\varepsilon}^{\alpha}+\varepsilon\right) p_{\varepsilon x} .
$$

By (15) the terms on the left hand side converge to the corresponding ones with $p_{\varepsilon}$ replaced by $p$. On the right hand side the term $\int_{0}^{t} \int_{\mathbb{R}} \varepsilon p_{\varepsilon x} \varphi_{x}$ vanishes as $\varepsilon \rightarrow 0$. The term $\int_{0}^{t} \int_{\mathbb{R}} \varphi_{x} \omega_{\varepsilon}^{\alpha} p_{\varepsilon x}$ can be rewritten as

$$
\int_{0}^{t} \int_{\mathbb{R}}\left(\left(1-\psi_{n}\right)+\psi_{n}\right) \varphi_{x} \omega_{\varepsilon}^{\alpha} p_{\varepsilon x}
$$

where $\psi_{n}$ is a smooth function such that $\operatorname{supp} \psi_{n} \subseteq\{\omega>0\}$ and $\psi_{n} \rightarrow \chi_{\{\omega>0\}}$ a.e. as $n \rightarrow \infty$. Therefore

$$
\int_{0}^{t} \int_{\mathbb{R}} \psi_{n} \varphi_{x} \omega_{\varepsilon}^{\alpha} p_{\varepsilon x} \stackrel{(\varepsilon \rightarrow 0)}{\longrightarrow} \int_{0}^{t} \int_{\mathbb{R}} \varphi_{x} \psi_{n} \omega^{\alpha} p_{x} \stackrel{(n \rightarrow \infty)}{\longrightarrow} \iint_{\{\omega>0\} \cap Q_{t}} \varphi_{x} \omega^{\alpha} p_{x},
$$

and, by 14,

$$
\begin{aligned}
\left|\int_{0}^{t} \int_{\mathbb{R}}\left(1-\psi_{n}\right) \varphi_{x} \omega_{\varepsilon}^{\alpha} p_{\varepsilon x}\right| & \leqslant C\left(\int_{0}^{t} \int_{\mathbb{R}} \omega_{\varepsilon}^{\alpha}\left(1-\psi_{n}\right)^{2}\right)^{1 / 2} \\
& \stackrel{(\varepsilon \rightarrow 0)}{\longrightarrow} C\left(\int_{0}^{t} \int_{\mathbb{R}} \omega^{\alpha}\left(1-\psi_{n}\right)^{2}\right)^{1 / 2} \stackrel{(n \rightarrow \infty)}{\longrightarrow} 0 .
\end{aligned}
$$

This completes the proof of the first integral identity of (iii).

The equation for $\omega_{\varepsilon}$ yields

$$
\begin{aligned}
\int_{\mathbb{R}} \varphi(t) \omega_{\varepsilon}(t)-\int_{\mathbb{R}} \varphi(0) \omega_{\varepsilon}(0)-\int_{0}^{t} \int_{\mathbb{R}} \varphi_{t} \omega_{\varepsilon}-A \int_{0}^{t} \int_{\mathbb{R}} \varphi\left(p_{\varepsilon}-I\right)_{+}^{\gamma}\left(1-\omega_{\varepsilon}\right) & \\
& =-\int_{0}^{t} \int_{\mathbb{R}} \varphi_{x}\left(\omega_{\varepsilon}^{\mu}\left(p_{\varepsilon}-I\right)_{+}^{\beta}+\varepsilon\right) \omega_{\varepsilon x} .
\end{aligned}
$$


Arguing as before, letting $\varepsilon \rightarrow 0$, we can replace $\omega_{\varepsilon}$ and $p_{\varepsilon}$ with $\omega$ and $p$ on the left hand side. The term on the right hand side can be rewritten as

$$
\int_{0}^{t} \int_{\mathbb{R}}\left(\left(1-\psi_{n}\right)+\psi_{n}\right) \varphi_{x}\left(\omega_{\varepsilon}^{\mu}\left(p_{\varepsilon}-I\right)_{+}^{\beta}+\varepsilon\right) \omega_{\varepsilon x},
$$

where $\psi_{n}$ is chosen as before. Again the terms containing the factor $1-\psi_{n}$ or $\varepsilon$ vanish as $\varepsilon \rightarrow 0$ and as $n \rightarrow \infty$. We consider the remaining term

$$
\begin{aligned}
\int_{0}^{t} \int_{\mathbb{R}} \psi_{n} \varphi_{x} \omega_{\varepsilon}^{\mu}\left(p_{\varepsilon}-I\right)_{+}^{\beta} \omega_{\varepsilon x}=I_{1}+I_{2} \\
\quad:=\iint_{\{p \geqslant I+\delta\} \cap Q_{t}} \psi_{n} \varphi_{x} \omega_{\varepsilon}^{\mu}\left(p_{\varepsilon}-I\right)_{+}^{\beta} \omega_{\varepsilon x}+\iint_{\{p<I+\delta\} \cap Q_{t}} \psi_{n} \varphi_{x} \omega_{\varepsilon}^{\mu}\left(p_{\varepsilon}-I\right)_{+}^{\beta} \omega_{\varepsilon x} .
\end{aligned}
$$

We observe that

$$
\begin{aligned}
\left|I_{2}\right| & \leqslant \iint_{\{p<I+\delta\} \cap Q_{t}}\left|\psi_{n} \varphi_{x} \omega_{\varepsilon}^{\mu}\left(p_{\varepsilon}-I\right)_{+}^{\beta} \omega_{\varepsilon x}\right| \leqslant C\left(\iint_{\{p<I+\delta\} \cap Q_{t}} \psi_{n}^{2} \varphi_{x}^{2} \omega_{\varepsilon}^{\mu}\left(p_{\varepsilon}-I\right)_{+}^{\beta}\right)^{1 / 2} \\
& \leqslant K \delta^{\beta / 2} \stackrel{(\delta \rightarrow 0)}{\longrightarrow} 0
\end{aligned}
$$

and

$$
\begin{gathered}
I_{1}=\iint_{\{p \geqslant I+\delta\} \cap Q_{t}} \psi_{n} \varphi_{x} \omega_{\varepsilon}^{\mu}\left(p_{\varepsilon}-I\right)_{+}^{\beta} \omega_{\varepsilon x} \stackrel{(\varepsilon \rightarrow 0)}{\longrightarrow} \iint_{\{p \geqslant I+\delta\} \cap Q_{t}} \psi_{n} \varphi_{x} \omega^{\mu}(p-I)_{+}^{\beta} \omega_{x} \\
\stackrel{(n \rightarrow \infty)}{\longrightarrow} \iint_{\{p \geqslant I+\delta\} \cap Q_{t}} \varphi_{x} \omega^{\mu}(p-I)_{+}^{\beta} \omega_{x} \stackrel{(\delta \rightarrow 0)}{\longrightarrow} \iint_{\{p>I\} \cap Q_{t}} \varphi_{x} \omega^{\mu}(p-I)_{+}^{\beta} \omega_{x} .
\end{gathered}
$$

Hence $(\omega, p)$ is a solution of Problem (1)-(2).

Part (i) of Theorem 2.3 follows at once from 16-(18). Part (ii) is a consequence of Proposition 3.1.iv):

$$
\left(\omega_{\varepsilon}\left(p_{\varepsilon}-I\right)_{+}\right)^{v} \in L^{2}\left((0, T) ; H^{1}(\mathbb{R})\right) \subseteq L^{2}((0, T) ; C(\mathbb{R})),
$$

i.e., the function $x \mapsto \omega(x, t)(p(x, t)-I)_{+}$is continuous for a.e. $t>0$. Finally, part (iii) follows from the following result, which we prove at the end of this section.

Proposition 5.2 If $p_{0} \geqslant I$, respectively $p_{0}>I$, a.e. in $(a, b)$, then for all $t>0, p(t) \geqslant I$, respectively $p(t)>I$, a.e. in $(a(t), b(t))$.

Proof of Proposition 5.1. Let $\underline{\omega}_{\varepsilon}$ be the solution of

$$
\begin{cases}z_{t}=\left(\left(\omega_{\varepsilon}^{\mu}\left(p_{\varepsilon}-I\right)_{+}^{\beta}+\varepsilon\right) z_{x}\right)_{x} & \text { in }(a, b) \times \mathbb{R}^{+} \\ z(x, 0)=\omega_{0}(x) & \text { for } x \in(a, b) \\ z(a, t)=z(b, t)=0 & \text { for } t>0\end{cases}
$$

Then, by the comparison principle,

$$
\omega_{\varepsilon} \geqslant \underline{\omega}_{\varepsilon} \quad \text { in }(a, b) \times \mathbb{R}^{+},
$$


and, up to subsequences, for all $T>0$,

$$
\underline{\omega}_{\varepsilon} \rightarrow \underline{\omega} \leqslant \omega \quad \text { in } C\left([0, T] ; L^{2}(a, b)\right)
$$

Arguing as in the proof for $\omega(x, t)$, we find that

$$
[a, b]=\operatorname{supp} \underline{\omega}\left(t_{1}\right)=\operatorname{supp} \underline{\omega}\left(t_{2}\right) \quad \text { if } 0 \leqslant t_{1} \leqslant t_{2} .
$$

We fix $T>0$. We divide the proof into several steps.

CLAIM 1 For all sufficiently small $\sigma>0$ there exists $\lambda_{\sigma}>0$ such that

$$
\omega_{\varepsilon} \geqslant \underline{\omega}_{\varepsilon} \geqslant \lambda_{\sigma} \quad \text { in }[a+\sigma, b-\sigma] \times[0, T] .
$$

Proof of Claim 1. By (37) we have, for $0 \leqslant t \leqslant T$,

$$
A_{\sigma}(t):=f_{[a, a+\sigma]} \underline{\omega}(t) \neq 0 \quad \text { and } \quad B_{\sigma}(t):=f_{[b-\sigma, b]} \underline{\omega}(t) \neq 0 .
$$

We define $A_{\sigma}^{\varepsilon}(t)$ and $B_{\sigma}^{\varepsilon}(t)$ in a similar way. Since $A_{\sigma}$ and $B_{\sigma}$ are continuous in $[0, T]$ there exists $\lambda_{\sigma}>0$ such that

$$
A_{\sigma}(t), B_{\sigma}(t) \geqslant 2 \lambda_{\sigma} \quad \text { for } 0 \leqslant t \leqslant T .
$$

Since $\underline{\omega}_{\varepsilon} \rightarrow \underline{\omega}$ in $C\left([0, T] ; L^{2}(a, b)\right)$,

$$
A_{\sigma}^{\varepsilon} \rightarrow A_{\sigma} \quad \text { and } \quad B_{\sigma}^{\varepsilon} \rightarrow B_{\sigma} \quad \text { uniformly in }[0, T]
$$

Hence, for $\varepsilon>0$ small enough,

$$
A_{\sigma}^{\varepsilon}(t), B_{\sigma}^{\varepsilon}(t)>\lambda_{\sigma} \quad \text { for } 0 \leqslant t \leqslant T .
$$

Now (38) follows from standard lap-number theory ([10]), where $\sigma$ is chosen so small that $\lambda_{\sigma}<\bar{\rho}$ ( $\bar{\rho}$ is defined by $(11)$ ).

REMARK 5.3 If $A=0$, we can apply the lap-number theory to the equation for $\omega_{\varepsilon}$ itself, and arguing as in the proof of Claim 1, with $A_{\sigma}$ replaced by $f_{[a(t), a(t)+\sigma]} \omega$, we obtain Proposition 5.1 So it remains to consider the case

$$
A>0 \text {. }
$$

Claim 2 Let $\left\{p_{0}>I\right\} \neq \emptyset$ and let $0<t_{0}<t_{1}$ and $x_{0}<x_{1}$ be such that

$$
K=\left[x_{0}, x_{1}\right] \times\left[t_{0}, t_{1}\right] \subseteq\{p>I\} .
$$

Then $w_{\varepsilon}$ and $p_{\varepsilon}$ are uniformly Hölder continuous in $K, \omega, p \in C(K)$ and there exists $\delta_{K}>0$ such that for all $\varepsilon>0$ small enough

$$
\omega_{\varepsilon} \geqslant \delta_{K} \quad \text { and } \quad p_{\varepsilon} \geqslant I+\delta_{K} \quad \text { in } K
$$




\section{REMARK 5.4}

(i) If $\left\{p_{0}>I\right\}=\emptyset$, then $p \leqslant I$ in $\mathbb{R} \times[0, \infty), \omega(x, t)=\omega_{0}(x)$ for all $x$ and $t$, and problem (1)- (2) becomes trivial.

(ii) As a consequence of Claim 2, $\omega_{\varepsilon}$ and $p_{\varepsilon}$ are uniformly Hölder continuous in any finite union of rectangles.

Proof of Claim 2. Since $\{p(t)>I+\rho\}$ is an interval if $0 \leqslant \rho<\bar{\rho}$ and $p \in C\left([0, \infty) ; L^{2}(\mathbb{R})\right)$, it follows that

$$
t \geqslant 0 \text { and } x \in\{p(t)>I\} \Rightarrow(x, t) \in\{p>I\} .
$$

Moreover, (40) holds with $\Rightarrow$ replaced by $\Leftrightarrow$. Indeed, let $(x, t) \in\{p>I\}$. Since the set $\{p>I\}$ is open and $p \in C\left([0, \infty) ; L_{\text {loc }}^{2}(\mathbb{R})\right)$, without loss of generality, we may assume that there exist $c, d \in \mathbb{R}^{+}$such that

$$
\begin{gathered}
{[x-c, x+c] \times[t-d, t+d] \subseteq\{p>I\},} \\
{[x-c, x+c] \subseteq\{p(s) \geqslant I\} \quad \text { for all } s \in(t-d, t+d]}
\end{gathered}
$$

and

$$
[x-c, x+c] \subseteq\left\{p(t-d)>I+\rho^{\prime}\right\}
$$

for some $\rho^{\prime}>0$. Arguing by contradiction we assume that $p(y, t)=I$ for all $y \in[x-c, x+c]$. Denote by $\left(\omega_{\varepsilon}, p_{\varepsilon}\right)$ the sequence defined by Theorem 3.2. By property 15 it follows that for each $\delta>0$,

$$
p_{\varepsilon}-I>-\delta \text { in }[x-c, x+c] \times[t-d, t+d],
$$

for $\varepsilon=\varepsilon(\delta)$ small enough; in particular,

$$
p_{\varepsilon}(y, t-d)>I+\rho^{\prime} / 2 \quad \text { in }[x-c, x+c] .
$$

Now, we proceed as in the proof of Lemma 4.1 . We multiply the equation for $p_{\varepsilon}$ by $-\phi^{2} /\left(\left(p_{\varepsilon}-I\right)+\right.$ $2 \delta$ ), where $\phi=\phi(y)$ is a Lipschitz continuous function with $\operatorname{supp} \phi \subseteq[x-c, x+c]$. Integrating by parts and letting $\varepsilon \rightarrow 0$, we find

$$
-\int_{\mathbb{R}} \phi^{2} \log (p-I+2 \delta)(t) \leqslant-\int_{\mathbb{R}} \phi^{2} \log (p-I+2 \delta)(t-d)+C(\phi) d \leqslant \bar{C} .
$$

Since $\delta$ is arbitrary, we have proved that

$$
t \geqslant 0 \text { and } x \in\{p(t)>I\} \Leftrightarrow(x, t) \in\{p>I\} .
$$

The set $\{p>I\}$ is connected, has a nonempty intersection with $(a, b) \times\{0\}$, and

$$
p(x, t) \leqslant I \quad \text { for all } x \in \complement \overline{\{p(t)>I\}}, t \geqslant 0 .
$$

Since $\{p>I\}$ is open there exists $\eta_{0} \in\left(0, t_{0}\right)$ such that for all $0 \leqslant \eta \leqslant \eta_{0}$,

$$
K_{\eta}:=\left[x_{0}-\eta, x_{1}+\eta\right] \times\left[t_{0}-\eta, t_{1}\right] \subseteq\{p>I\} .
$$

Arguing as in the proof of Claim 1 it follows that for all $0 \leqslant \eta<\eta_{0}$ there exists $\delta(\eta)$ such that

$$
p_{\varepsilon} \geqslant \delta(\eta)>0 \quad \text { in } K_{\eta},
$$

for $\varepsilon$ sufficiently small. Hence, by [8], $\omega_{\varepsilon}$, and therefore $\omega$, are equicontinuous in $K_{\eta}$ for $0 \leqslant \eta<\eta_{0}$. 
It remains to prove that $\omega>0$ in $K$. Arguing by contradiction, assume that $\omega(\bar{x}, \bar{t})=0$ for some $(\bar{x}, \bar{t}) \in K$. We choose $\eta \in\left(0, \eta_{0}\right)$. Since $\left[x_{1}-\eta, x_{2}+\eta\right] \subseteq \operatorname{supp} p(\bar{t})=\operatorname{supp} \omega(\bar{t})$, there exist $x^{-} \in\left(x_{1}-\eta, \bar{x}\right)$ and $x^{+} \in\left(\bar{x}, x_{2}+\eta\right)$ such that $\omega\left(x^{ \pm}, t\right)>0$. By the continuity of $\omega$ there exists $t^{-} \in\left(t_{0}-\eta, \bar{t}\right)$ such that

$$
\omega\left(x^{ \pm}, t\right)>C_{0}>0 \quad \text { for } t^{-} \leqslant t \leqslant \bar{t} .
$$

Since $A>0$ and $p \geqslant \delta(\eta)>0$, the comparison principle shows that

$$
\omega(x, t) \geqslant C_{1}\left(t-t^{-}\right) \quad \text { for } x^{-} \leqslant x \leqslant x^{+}, t^{-} \leqslant t \leqslant \bar{t}
$$

where $C_{1}>0$ is a sufficiently small constant. Hence $\omega(\bar{x}, \bar{t}) \geqslant C_{1}\left(\bar{t}-t^{-}\right)>0$ and we have found a contradiction.

Now we are ready to complete the proof of Proposition 5.1 We argue by contradiction and suppose that $\omega\left(x_{0}, t_{0}\right)=0$ for some $t_{0}>0$. By Claim 1 , we can assume $x_{0} \geqslant b$ (the case $x_{0} \leqslant a$ is completely similar). Since $b(t)$ is continuous and $b\left(t_{0}\right)>x_{0} \geqslant b=b(0)$, we have

$$
\tau:=\inf \left\{t>0: b(t)>x_{0}\right\}>0 \quad \text { and } \quad x_{0}=b(\tau) .
$$

We claim that

$$
\{p>I\} \cap \Lambda\left(\tau, t_{0}\right) \neq \emptyset,
$$

where we have set

$$
\Lambda\left(\tau, t_{0}\right):=\left\{(x, t): x_{0}<x<b(t), \tau<t<t_{0}\right\} .
$$

Indeed, otherwise $\omega=0$ in $\Lambda\left(\tau, t_{0}\right)$, and since $[a(t), b(t)]=\operatorname{supp} \omega(t)$ we obtain a contradiction.

Let $\left(x_{1}, t_{1}\right) \in\{p>I\} \cap \Lambda\left(\tau, t_{0}\right)$. From the connectedness of $\{p>I\}$, it follows that there exist $\left(\eta_{1}, \tau_{1}\right) \in\{p>I\}$ and a smooth function $g(t)\left(\tau_{1} \leqslant t \leqslant t_{1}\right)$ such that

$$
\begin{gathered}
0 \leqslant \tau_{1}<t_{0}, \quad \eta_{1} \in(a, b), \quad g\left(\tau_{1}\right)=\eta_{1}, \quad g\left(t_{1}\right)=x_{1}, \\
(g(t), t) \in\{p>I\}, \quad g(t)>a \quad \text { if } \tau_{1} \leqslant t \leqslant t_{1} .
\end{gathered}
$$

Let $x^{-}>a$ be such that $x^{-}<g(t)$ for all $\tau_{1} \leqslant t \leqslant t_{1}$. By Claims 1 and 2 there exists $C>0$ such that

$$
\omega(x, t) \geqslant 2 C>C
$$

if $(x, t)$ satisfies one of the following conditions:

(i) $x=x^{-}, \tau_{1} \leqslant t \leqslant t_{1}$;

(ii) $t=\tau_{1}, x^{-}<x<\eta_{1}=g\left(\tau_{1}\right)$;

(iii) $x=g(t), \tau_{1} \leqslant t \leqslant t_{1}$.

Observing that

$$
E:=\left\{(g(t), t): t \in\left[\tau_{1}, t_{1}\right]\right\}
$$

is compact, we can find a finite number of sets of the form

$$
K_{j}:=\left[\underline{x}_{j}, \bar{x}_{j}\right] \times\left[\underline{t}_{j}, \bar{t}_{j}\right], \quad j=1, \ldots, n,
$$

such that

$$
E \subset \widetilde{K}:=\bigcup_{j=1}^{n} K_{j} \subseteq\{p>I\}
$$


and $\omega_{\varepsilon} \geqslant C$ in $\widetilde{K}$ for all $\varepsilon$ sufficiently small. In addition, by Claim $1, \omega_{\varepsilon}(x, t) \geqslant C$ for $\varepsilon$ small enough, if $(x, t)$ satisfies conditions (i) and (iii). Hence, by the maximum principle,

$$
\omega_{\varepsilon}(x, t), \omega(x, t) \geqslant C \quad \text { if } x^{-} \leqslant x \leqslant g(t), \tau_{1} \leqslant t \leqslant t_{1} .
$$

Arguing as in Claim 1, with $(a, b) \times(0, T)$ replaced by $\left(x^{-}, x_{1}\right) \times\left(t, t_{0}\right)$, we conclude that $\omega_{\varepsilon}\left(x_{0}, t_{0}\right), \omega\left(x_{0}, t_{0}\right)>C^{\prime}$ and we have found a contradiction.

Proof of Proposition 5.2 First we consider the case $p_{0} \geqslant I$ in $(a, b)$. We assume $\left\{p_{0}>I\right\} \neq \emptyset$, otherwise the result is trivial. We argue by contradiction and assume that the set $\{p<I\} \cap\{\omega>0\}$ is not empty. Define

$$
\tau=\sup \{t:\{p(t)>I\} \neq \emptyset\} \quad(\text { possibly } \tau=+\infty) .
$$

We dinstinguish two different cases:

CASE 1 There exists $\left(x_{1}, t_{1}\right) \in\{p<I\} \cap\{\omega>0\}$ such that $t_{1} \leqslant \tau$.

CASE 2 The set $\{p<I\} \cap\{\omega>0\} \cap([0, \tau] \times \mathbb{R})$ is empty.

Case 1. Since $\{p(t) \geqslant I\}$ is an interval and $p \in C\left([0, T] ; L_{\mathrm{loc}}^{2}(\mathbb{R})\right)$, without loss of generality, we may suppose that there exist $\delta>0$ and $x_{2} \geqslant x_{1}$ such that

$$
p<I \quad \text { in }\left[x_{2}, \infty\right) \times\left[t_{1}-\delta, t_{1}+\delta\right],
$$

and

$$
x_{2}<b(t) \quad \text { for } t_{1}-\delta \leqslant t \leqslant t_{1}+\delta .
$$

As a consequence, by [19], $b(t)=b\left(t_{1}\right)$ for all $t \in\left[t_{1}-\delta, t_{1}+\delta\right]$. Let

$$
\begin{array}{r}
t_{0}:=\inf \left\{t \leqslant t_{1}: \text { for all } t \leqslant s \leqslant t_{1} \text { there exists } x_{s} \in(a(s), b(s))\right. \\
\text { such that } \left.p(x, s)<I \text { for all } x \geqslant x_{s}\right\} .
\end{array}
$$

Then $b(t)=b\left(t_{0}\right)$ for $t_{0} \leqslant t \leqslant t_{1}$. By construction, there exists $x_{0} \in(a(t), b(t))$ such that

$$
p\left(x, t_{0}\right) \geqslant I \quad \text { in }\left(x_{0}, b\left(t_{0}\right)\right) .
$$

Let us still denote by $\left(x_{0}, b\left(t_{0}\right)\right)$ the maximal interval for which (42) is satisfied. Since $\tau>t_{0}$ we can assume that

$$
\left(y_{0}, t_{0}\right) \in\{p>I\}
$$

for some $y_{0} \in\left(x_{0}, b\left(t_{0}\right)\right)$. On the other hand $\{p>I\}$ is open, therefore there exists $\delta_{0}>0$ such that

$$
p>I \quad \text { in }\left[y_{0}-\delta_{0}, y_{0}+\delta_{0}\right] \times\left[t_{0}, t_{0}+\delta\right] .
$$

Now let $\varphi \in \operatorname{Lip}(\mathbb{R})$ be such that $0 \leqslant \varphi \leqslant 1$ and

$$
\varphi(x)= \begin{cases}1 & \text { if } x \in\left[y_{0}+\delta_{0}, b\left(t_{0}\right)\right] \\ 0 & \text { if } x \in\left(-\infty, y_{0}-\delta_{0}\right] \cup\left[b\left(t_{0}\right)+\delta, \infty\right) .\end{cases}
$$

Testing the equation for $p_{\varepsilon}$ with $\varphi^{2}\left(p_{\varepsilon}-I\right)_{-}$in $\mathbb{R} \times\left[t_{0}, t_{0}+\delta_{0}\right]$, we find

$$
\begin{aligned}
-\frac{\mathrm{d}}{\mathrm{d} t} \int_{\mathbb{R}} \frac{1}{2} \varphi^{2}\left(p_{\varepsilon}-I\right)_{-}^{2}= & 2 \int_{\mathbb{R}}\left(\omega_{\varepsilon}^{\alpha}+\varepsilon\right)\left(p_{\varepsilon}-I\right)_{-}\left[\left(p_{\varepsilon}-I\right)_{-}\right]_{x} \varphi \varphi_{x} \\
& +\int_{\mathbb{R}}\left[\left(p_{\varepsilon}-I\right)_{-}\right]_{x}^{2}\left(\omega_{\varepsilon}^{\alpha}+\varepsilon\right) \varphi^{2} \\
\geqslant & -C \int_{\mathbb{R}}\left[\left(p_{\varepsilon}-I\right)_{-}\right]^{2}\left(\omega_{\varepsilon}^{\alpha}+\varepsilon\right) \varphi_{x}^{2},
\end{aligned}
$$


where the last term vanishes as $\varepsilon \rightarrow 0$. Since $\int_{b\left(t_{0}\right)}^{\infty}(p-I)_{-} \varphi^{2}$ is constant, this yields

$$
\int_{y_{0}+\delta_{0}}^{b\left(t_{0}\right)}(p-I)_{-}^{2}(t) \leqslant \int_{y_{0}+\delta_{0}}^{b\left(t_{0}\right)} \varphi^{2}(p-I)_{-}^{2}\left(t_{0}\right)=0 \quad \text { for } t \in\left(t_{0}, t_{0}+\delta_{0}\right] .
$$

In view of the definition of $t_{0}$, we have found a contradiction.

Case 2. We have

$$
p \geqslant I \quad \text { in }\{\omega>0\} \cap Q_{\tau}, \quad p(\tau)=I \quad \text { in }(a(\tau), b(\tau)), \quad p(t) \leqslant I \quad \text { for } t>\tau .
$$

Therefore, for all $t>\tau$ we have

$$
\omega(x, t)=\omega(x, \tau) \quad \text { for } x \in \mathbb{R},
$$

and

$$
a(t)=a(\tau), \quad b(t)=b(\tau) .
$$

Since

$$
\int_{a(\tau)}^{b(\tau)} p(t)=\int_{a(\tau)}^{b(\tau)} p(\tau)=I(b(\tau)-a(\tau))
$$

we conclude that

$$
p=I \quad \text { in }(a(\tau), b(\tau)) \times[\tau, \infty),
$$

and we have found a contradiction.

Finally, consider the case $p_{0}>I$ in $(a, b)$. Then $p \geqslant I$ in $\{\omega>0\}$ and arguing as in the proof of 41 we obtain the result easily.

\section{Appendix A. Finite speed of propagation}

This section is devoted to the proof of Theorem 4.4. Our approach will use the techniques originally developed in [6, 7] to establish the occurrence of waiting time phenomena for the thin film equation as well as for higher order doubly nonlinear equations with variational structure. Therefore the main tool will be to derive a suitable integral inequality, to which we can apply Stampacchia's iteration lemma [14, Lemma 4.1]:

LEMmA A.1 Assume that a given nonnegative nonincreasing function $G:\left[0, \xi_{0}\right] \rightarrow \mathbb{R}$ satisfies

$$
G(\xi) \leqslant \frac{c_{0}}{(\xi-\eta)^{\lambda}} G(\eta)^{1+\kappa}
$$

for $0 \leqslant \eta \leqslant \xi \leqslant \xi_{0}$ and positive numbers $c_{0}, \lambda$ and $\kappa$. If

$$
\xi_{0}^{\lambda} \geqslant 2^{\lambda(1+\kappa) / \kappa} c_{0} G(0)^{\kappa},
$$

then $G\left(\xi_{0}\right)=0$.

In the proof we will use the Gagliardo-Nirenberg inequality. In the version stated below, some of the summability powers are allowed to be less than one (see [6] for a more general statement). 
THEOREM A.2 (Gagliardo-Nirenberg) Let $0<s<p, 1 \leqslant r \leqslant \infty, n \in \mathbb{N}, n>0,0 \leqslant \theta<1$. Let $\Omega \subseteq \mathbb{R}^{N}$ be open and diffeomorphic to a cone. Suppose that $u \in L^{s}(\Omega)$ and its derivative of order $n$ belongs to $L^{r}(\Omega)$. Then the following inequality holds (with a constant $K$ depending only on $N, n, r, s$ and $\Omega$ ):

$$
\|u\|_{p} \leqslant K\left\|D^{n} u\right\|_{r}^{\theta}\|u\|_{s}^{1-\theta}
$$

if

$$
\frac{1}{p}=\theta\left(\frac{1}{r}-\frac{n}{N}\right)+(1-\theta) \frac{1}{s} .
$$

Proof of Theorem 4.4 First we will derive the integral inequality. Let $\varepsilon>0$ and let $\left(\omega_{\varepsilon}, p_{\varepsilon}\right)$ be the solution of Problem (12) defined by Proposition 3.1. For a compactly supported function $\varphi \in W^{1, \infty}(\mathbb{R})$, we set

$$
\Phi:=\varphi^{m}\left(p_{\varepsilon}-I\right)_{+}^{\delta}, \quad \Psi:=\varphi^{m} \omega_{\varepsilon}^{\sigma} .
$$

We keep the possibility to choose later the constants $\delta, \sigma, m \in \mathbb{R}^{+}, m \geqslant 2$. Taking $\Phi$ (resp. $\Psi$ ), as test function for the equation for $p_{\varepsilon}$ (resp. $\omega_{\varepsilon}$ ), and integrating by parts in $\mathbb{R} \times[0, t], t \leqslant T$, we obtain

$$
\begin{aligned}
& \int_{\mathbb{R}} \varphi^{m} \frac{\left(p_{\varepsilon}-I\right)_{+}^{\delta+1}}{\delta+1}(t)-\int_{\mathbb{R}} \varphi^{m} \frac{\left(p_{0}-I\right)_{+}^{\delta+1}}{\delta+1} \\
& =-m \int_{0}^{t} \int_{\mathbb{R}} \varphi^{m-1} \varphi_{x}\left(\omega_{\varepsilon}^{\alpha}+\varepsilon\right)\left(p_{\varepsilon}-I\right)_{+}^{\delta} p_{\varepsilon x}-\delta \int_{0}^{t} \int_{\mathbb{R}} \varphi^{m}\left(\omega_{\varepsilon}^{\alpha}+\varepsilon\right)\left(p_{\varepsilon}-I\right)_{+}^{\delta-1}\left(p_{\varepsilon x}\right)^{2}
\end{aligned}
$$

and

$$
\begin{aligned}
\int_{\mathbb{R}} \varphi^{m} & \frac{\omega_{\varepsilon}^{\sigma+1}}{\sigma+1}(t)-\int_{\mathbb{R}} \varphi^{m} \frac{\omega_{0}^{\sigma+1}}{\sigma+1}=-m \int_{0}^{t} \int_{\mathbb{R}} \varphi^{m-1} \varphi_{x}\left(\omega_{\varepsilon}^{\mu}\left(p_{\varepsilon}-I\right)_{+}^{\beta}+\varepsilon\right) \omega_{\varepsilon}^{\sigma} \omega_{\varepsilon x} \\
& -\sigma \int_{0}^{t} \int_{\mathbb{R}} \varphi^{m}\left(\omega_{\varepsilon}^{\mu}\left(p_{\varepsilon}-I\right)_{+}^{\beta}+\varepsilon\right) \omega_{\varepsilon}^{\sigma-1}\left(\omega_{\varepsilon x}\right)^{2}+A \int_{0}^{t} \int_{\mathbb{R}} \varphi^{m} \omega_{\varepsilon}^{\sigma}\left(p_{\varepsilon}-I\right)_{+}^{\gamma}\left(1-\omega_{\varepsilon}\right) .
\end{aligned}
$$

Adding 467 and 477 and applying Hölder's and Young's inequalities, we find

$$
\begin{aligned}
\int_{\mathbb{R}} \varphi^{m}\left(\frac{\left(p_{\varepsilon}-I\right)_{+}^{\delta+1}}{\delta+}\right. & \left.(t)+\frac{\omega_{\varepsilon}^{\sigma+1}}{\sigma+1}(t)\right) \leqslant \int_{\mathbb{R}} \varphi^{m}\left(\frac{\left(p_{0}-I\right)_{+}^{\delta+1}}{\delta+1}+\frac{\omega_{0}^{\sigma+1}}{\sigma+1}\right) \\
& +C_{1} \int_{0}^{t} \int_{\mathbb{R}} \varphi^{m-2} \varphi_{x}^{2}\left(\left(\omega_{\varepsilon}^{\alpha}+\varepsilon\right)\left(p_{\varepsilon}-I\right)_{+}^{\delta+1}+\omega_{\varepsilon}^{\mu}\left(\left(p_{\varepsilon}-I\right)_{+}^{\beta}+\varepsilon\right) \omega_{\varepsilon}^{\sigma+1}\right) \\
& -C_{2} \int_{0}^{t} \int_{\mathbb{R}} \varphi^{m}\left(\left(\omega_{\varepsilon}^{\alpha}+\varepsilon\right)\left(p_{\varepsilon}-I\right)_{+}^{\delta-1} p_{\varepsilon x}^{2}+\left(\omega_{\varepsilon}^{\mu}\left(p_{\varepsilon}-I\right)_{+}^{\beta}+\varepsilon\right) \omega_{\varepsilon}^{\sigma-1} \omega_{\varepsilon x}^{2}\right) \\
& +C_{3} \int_{0}^{t} \int_{\mathbb{R}} \varphi^{m} \omega_{\varepsilon}^{\sigma}\left(p_{\varepsilon}-I\right)_{+}^{\gamma}=: I_{0}+C_{1} I_{1}-C_{2} I_{2}+C_{3} I_{3},
\end{aligned}
$$

where the constants $C_{1}, C_{2}, C_{3}$ depend only on $m, \delta, \sigma$ and $A$. Letting $\bar{\beta}:=\min \{\beta, \alpha \gamma\}$, we choose $\delta$ and $\sigma$ such that

$$
\sigma+1>\alpha, \quad \delta+1>\max \{\bar{\beta}, \gamma\} .
$$


To estimate $I_{1}$, we rewrite

$$
\begin{aligned}
I_{1}= & \int_{0}^{t} \int_{\mathbb{R}} \varphi^{m-2} \varphi_{x}^{2}\left(\omega_{\varepsilon}^{\alpha}\left(p_{\varepsilon}-I\right)_{+}^{\delta+1}+\omega_{\varepsilon}^{\mu+\sigma+1}\left(p_{\varepsilon}-I\right)_{+}^{\beta}\right) \\
& +\varepsilon \int_{0}^{t} \int_{\mathbb{R}} \varphi^{m-2} \varphi_{x}^{2}\left(\left(p_{\varepsilon}-I\right)_{+}^{\delta+1}+\omega_{\varepsilon}^{\mu+\sigma+1}\right)=: I_{1}^{1}+I_{1}^{2}
\end{aligned}
$$

and we observe that

$$
I_{1}^{2} \rightarrow 0 \quad \text { as } \varepsilon \rightarrow 0
$$

Hence

$$
I_{1}^{1}=\int_{0}^{t} \int_{\mathbb{R}} \varphi^{m-2} \varphi_{x}^{2} \omega_{\varepsilon}^{\alpha}\left(p_{\varepsilon}-I\right)_{+}^{\bar{\beta}}\left(\left(p_{\varepsilon}-I\right)_{+}^{\delta+1-\bar{\beta}}+\omega_{\varepsilon}^{\mu+\sigma+1-\alpha}\left(p_{\varepsilon}-I\right)_{+}^{\beta-\bar{\beta}}\right),
$$

and from Hölder's and Young's inequalities it follows that

$$
\begin{aligned}
I_{1}^{1} \leqslant & \frac{q-1}{q} \int_{0}^{t} \int_{\mathbb{R}} \varphi^{(m-2) \frac{q}{q-1}}\left(\left(p_{\varepsilon}-I\right)_{+}^{(\delta+1-\bar{\beta}) \frac{q}{q-1}}+\omega_{\varepsilon}^{(\mu+\sigma+1-\alpha) \frac{q}{q-1}}\right) \\
& +\frac{\left\|\varphi_{x}\right\|_{\infty}}{q} \int_{0}^{t} \int_{\operatorname{supp} \varphi} \omega_{\varepsilon}^{\alpha q}\left(p_{\varepsilon}-I\right)_{+}^{\bar{\beta} q},
\end{aligned}
$$

where $q>1$. Choosing $\delta, \sigma$ and $m$ such that

$$
\begin{array}{ll}
\text { (i) } \frac{\delta+1}{\bar{\beta}}=q=\frac{\sigma+1}{\alpha}, & \text { (ii) } m=2 q,
\end{array}
$$

we obtain

$$
I_{1}^{1} \leqslant C_{4} \int_{0}^{t} \int_{\mathbb{R}} \varphi^{m}\left(\left(p_{\varepsilon}-I\right)_{+}^{\delta+1}+\omega_{\varepsilon}^{\sigma+1}\right)+C_{5} \int_{0}^{t} \int_{\operatorname{supp} \varphi} \omega_{\varepsilon}^{\sigma+1}\left(p_{\varepsilon}-I\right)_{+}^{\delta+1} .
$$

To handle $I_{2}$ we have to determine $l \in \mathbb{R}^{+}$such that

$$
\left[\left(\omega_{\varepsilon}^{\sigma+1}\left(p_{\varepsilon}-I\right)_{+}^{\delta+1}\right)^{l}\right]_{x}^{2} \leqslant C_{6}\left(\omega_{\varepsilon}^{\alpha}\left(p_{\varepsilon}-I\right)_{+}^{\delta-1} p_{\varepsilon x}^{2}+\omega_{\varepsilon}^{\mu+\sigma+1}\left(p_{\varepsilon}-I\right)_{+}^{\beta} \omega_{\varepsilon x}^{2}\right) .
$$

A straightforward computation leads to the condition

$$
l \geqslant \max \left\{\frac{\mu+\sigma+1}{2(\sigma+1)}, \frac{\beta}{2(\delta+1)}\right\} .
$$

As a consequence,

$$
I_{2} \geqslant C_{7} \int_{0}^{t} \int_{\mathbb{R}} \varphi^{m}\left[\left(\omega_{\varepsilon}^{\sigma+1}\left(p_{\varepsilon}-I\right)_{+}^{\delta+1}\right)^{l}\right]_{x}^{2} .
$$

Finally, we estimate $I_{3}$. By Hölder's and Young's inequalities, we get

$$
I_{3} \leqslant C_{8} \int_{0}^{t} \int_{\mathbb{R}} \varphi^{m}\left(\omega_{\varepsilon}^{\frac{\delta+1}{\delta+1-\gamma}}+\left(p_{\varepsilon}-I\right)_{+}^{\delta+1}\right) .
$$


It follows from assumption 5 (50) (i) that $\sigma+1 \geqslant(\delta+1) / \gamma$. Hence $\sigma(\delta+1) /(\delta+1-\gamma) \geqslant \sigma+1$, and

$$
I_{3} \leqslant C_{8} \int_{0}^{t} \int_{\mathbb{R}} \varphi^{m}\left(\left(p_{\varepsilon}-I\right)_{+}^{\delta+1}+\omega_{\varepsilon}^{\sigma+1}\right) .
$$

Combining (51), 53) and (54) and letting $\varepsilon \rightarrow 0$, we obtain

$$
\begin{aligned}
\int_{\mathbb{R}} \varphi^{m}\left(\omega^{\sigma+1}+\right. & \left.(p-I)_{+}^{\delta+1}\right)(t)+C_{9} \int_{0}^{t} \int_{\mathbb{R}} \varphi^{m}\left[\left(\omega^{\sigma+1}(p-I)_{+}^{\delta+1}\right)^{l}\right]_{x}^{2} \\
\leqslant & C_{10} \int_{\mathbb{R}} \varphi^{m}\left(\omega_{0}^{\sigma+1}+\left(p_{0}-I\right)_{+}^{\delta+1}\right)+C_{11} \int_{0}^{t} \int_{\mathbb{R}} \varphi^{m}\left(\omega^{\sigma+1}+(p-I)_{+}^{\delta+1}\right) \\
& +C_{12} \int_{0}^{t} \int_{\operatorname{supp} \varphi} \omega^{\sigma+1}(p-I)_{+}^{\delta+1} \\
\leqslant & C_{10} \int_{\mathbb{R}} \varphi^{m}\left(\omega_{0}^{\sigma+1}+\left(p_{0}-I\right)_{+}^{\delta+1}\right)+C_{11} t \sup _{\tau} \int_{\mathbb{R}} \varphi^{m}\left(\omega^{\sigma+1}+(p-I)_{+}^{\delta+1}\right) \\
& +C_{12} \int_{0}^{t} \int_{\operatorname{supp} \varphi} \omega^{\sigma+1}(p-I)_{+}^{\delta+1}
\end{aligned}
$$

for all $0<t<\tau \leqslant T$. Taking the supremum in $(0, \tau)$ on the left hand side and imposing $C_{11} \tau \leqslant$ $1 / 2$, we find

$$
\begin{aligned}
\sup _{t \in(0, \tau)} \int_{\mathbb{R}} \varphi^{m}\left(\omega^{\sigma+1}+(p-I)_{+}^{\delta+1}\right)(t)+C_{9} \int_{0}^{\tau} \int_{\mathbb{R}} \varphi^{m}\left[\left(\omega^{\sigma+1}(p-I)_{+}^{\delta+1}\right)^{l}\right]_{x}^{2} \\
\leqslant C_{10}^{\prime} \int_{\mathbb{R}} \varphi^{m}\left(\omega_{0}^{\sigma+1}+\left(p_{0}-I\right)_{+}^{\delta+1}\right)+C_{12}^{\prime} \int_{0}^{\tau} \int_{\operatorname{supp} \varphi} \omega^{\sigma+1}(p-I)_{+}^{\delta+1} .
\end{aligned}
$$

Finally, defining

$$
w=\left(\omega^{(\sigma+1) / 2}(p-I)_{+}^{(\delta+1) / 2}\right)^{l^{\prime}}, \quad l^{\prime}=2 l,
$$

it follows from 55 that for some positive constant $\bar{C}$,

$$
\begin{aligned}
\sup _{t \in(0, \tau)} \int_{\mathbb{R}} \varphi^{m} w^{1 / l^{\prime}}(t)+\bar{C}^{-1} \int_{0}^{\tau} & \int_{\mathbb{R}} \varphi^{m} w_{x}^{2} \\
& \leqslant \bar{C} \int_{\mathbb{R}} \varphi^{m}\left(\omega_{0}^{\sigma+1}+\left(p_{0}-I\right)_{+}^{\delta+1}\right)+\bar{C} \int_{0}^{\tau} \int_{\operatorname{supp} \varphi} w^{2 / l^{\prime}},
\end{aligned}
$$

which is the desired integral inequality.

Since $\omega, p \in L_{\mathrm{loc}}^{\infty}\left([0, \infty) ; L^{q}(\mathbb{R})\right)$ for all $1 \leqslant q<\infty$, we observe that $(56)$ is still satisfied for all $\varphi \in W^{1, \infty}(\mathbb{R})$.

Without loss of generality, we may assume that $a=\min \operatorname{supp} \omega_{0}=1$. For a positive number $r<1$ we take

$$
\varphi=\varphi_{r}(x):= \begin{cases}1 & \text { if } x<r-1, \\ r-x & \text { if } x \in[r-1, r], \\ 0 & \text { otherwise. }\end{cases}
$$


Thus, for arbitrary $0<\rho<r, 56$ can be rewritten in the following way:

$$
\sup _{t \in(0, \tau)} \int_{-\infty}^{\rho} w^{1 / l^{\prime}}+\bar{C}^{-1} \int_{0}^{\tau} \int_{-\infty}^{\rho} w_{x}^{2} \leqslant \frac{\bar{C}}{(r-\rho)^{m}} \int_{0}^{\tau} \int_{-\infty}^{r} w^{2 / l^{\prime}} .
$$

We estimate $\int_{0}^{\tau} \int_{-\infty}^{\rho} w^{2 / l^{\prime}}$ by Gagliardo-Nirenberg's inequality (cf. Theorem A.2):

$$
\left(\int_{-\infty}^{\rho} w^{2 / l^{\prime}}\right)^{l^{\prime} / 2} \leqslant K\left(\int_{-\infty}^{\rho} w_{x}^{2}\right)^{\theta / 2}\left(\int_{-\infty}^{\rho} w^{1 / l^{\prime}}\right)^{l^{\prime}(1-\theta)}
$$

with

$$
\theta=\frac{l^{\prime}}{1+2 l^{\prime}}<1
$$

As a consequence,

$$
\begin{aligned}
\int_{0}^{\tau} \int_{-\infty}^{\rho} w^{2 / l^{\prime}} & \leqslant K_{1} \int_{0}^{\tau}\left(\int_{-\infty}^{\rho} w_{x}^{2}\right)^{\frac{1}{1+2 l^{\prime}}}\left(\int_{-\infty}^{\rho} w^{\frac{1}{l^{\prime}}}\right)^{2 \frac{1+l^{\prime}}{1+2 l^{\prime}}} \\
& \left.\leqslant K_{1}\left(\int_{0}^{\tau} \int_{-\infty}^{\rho} w_{x}^{2}\right)^{\frac{1}{1+2 l^{\prime}}}\left(\int_{0}^{\tau}\left(\int_{-\infty}^{\rho} w^{\frac{1}{l^{\prime}}}\right)^{2 \frac{1+l^{\prime}}{1+2 l^{\prime}}}\right)^{\frac{1+2 l^{\prime}}{2 l^{\prime}}}\right)^{\frac{2 l^{\prime}}{1+2 l^{\prime}}} \\
& \leqslant K_{1}\left(\int_{0}^{\tau} \int_{-\infty}^{\rho} w_{x}^{2}\right)^{\frac{1}{1+2 l^{\prime}}} \tau^{\frac{2 l^{\prime}}{1+2 l^{\prime}}}\left(\sup _{(0, \tau)}^{\rho} \int_{-\infty}^{\frac{1}{l^{\prime}}}\right)^{\frac{1+l^{\prime}}{1+2 l^{\prime}}}
\end{aligned}
$$

Combining this with 57 yields

$$
\int_{0}^{\tau} \int_{-\infty}^{\rho} w^{2 / l^{\prime}} \leqslant K_{2} \tau^{\frac{2 l^{\prime}}{1+2 l^{\prime}}}\left(\frac{1}{(r-\rho)^{m}} \int_{0}^{\tau} \int_{-\infty}^{r} w^{2 / l^{\prime}}\right)^{1+\frac{2}{1+2 l^{\prime}}} .
$$

Let us introduce for $\xi \geqslant 0$ the decreasing nonnegative function

$$
G(\xi):=\int_{0}^{\tau} \int_{-\infty}^{1-\xi} w^{2 / l^{\prime}}
$$

Hence, for $\xi=1-\rho$ and $\eta=1-r$, 58 becomes

$$
G(\xi) \leqslant K_{2} \frac{\tau^{\frac{2 l^{\prime}}{1+2 l^{\prime}}}}{(\xi-\eta)^{m(1+\kappa)}} G(\eta)^{1+\kappa}
$$

for $0 \leqslant \eta \leqslant \xi \leqslant 1, \kappa=2 /\left(1+2 l^{\prime}\right)$. Applying Stampacchia's Lemma A.1. we find $G\left(\xi_{0}\right)=0$ provided

$$
\xi_{0}^{m(1+\kappa)} \geqslant 2^{m(1+\kappa)^{2} / \kappa} K_{2} \tau^{(2-\kappa) / 2} G(0)^{\kappa} .
$$

Since $G(0) \leqslant \tau\left\|p_{0}\right\|_{1}$, 59, is satisfied if

$$
\xi_{0} \geqslant K_{3} \tau^{\frac{2+\kappa}{2 m(1+\kappa)}}=: 1-\rho_{0}(\tau)
$$


Thus, for sufficiently small $\tau^{*}=\tau^{*}\left(K_{3}\right)$, from 60 we conclude that

$$
\int_{0}^{t} \int_{-\infty}^{\rho_{0}(t)} \omega^{\sigma+1}(p-I)_{+}^{\delta+1}=0
$$

for all $0 \leqslant t \leqslant \tau^{*}$. This yields $\omega(x, t)=0$ for all $x<\rho_{0}(t), 0 \leqslant t \leqslant \tau^{*}$, i.e.

$$
r(t)=K_{3} \frac{2+\kappa}{2 m(1+\kappa)}, \quad 0 \leqslant t \leqslant \tau^{*} .
$$

Since the constants involved in the proof are independent of time the proof of the theorem is complete.

\section{Conclusions}

The system we consider is motivated by the study of subterranean fluid flows in the framework of nonlocal continuum damage mechanics, according to a model which has recently been introduced by Barenblatt, Patzek, Prostokishin and Silin [3]. In particular they conjectured the existence of a free moving boundary of the damaged region. The system is nonstandard and, even after a slight simplification explained in the introduction, the definition and the existence of solutions turn out to be nontrivial problems. In fact both the pressure $p$ and the damage $\omega$ may become discontinuous across the free boundary, as was pointed out in [5]. Therefore a detailed analysis of the support properties is essential in our study.

One of the main open problems is to extend our analysis to higher space dimension, since even the validity of the basic BV estimates, which play an essential role in the compactness results, is not clear. The problem of uniqueness of solutions is completely open, even in the case of one space dimension.

\section{Acknowledgments}

The authors express sincere gratitude to Prof. G. I. Barenblatt for suggesting the problem and for many stimulating discussions.

The third author was supported in part by the Director, Office of Science, Computational and Technology Research, U.S. Department of Energy under Contract No. DE-AC03-76SF00098. He thanks the Lawrence Berkeley National Laboratory, where he carried out most of his research, for the great hospitality.

\section{REFERENCES}

1. Amann, H. Dynamic theory of quasilinear parabolic equations III. Global existence. Math. Z. 202 (1989), 219-250. Zbl 0702.35125 MR 1013086

2. Barenblatt, G. I., Bertsch, M., \& Nitsch, C. Nonlocal damage accumulation and fluid flow in diatomites. Preprint (2005).

3. Barenblatt, G. I., Patzek, T. W., Prostokishin, V. M., \& Silin, D. B. Oil deposit in diatomites: a new challenge for subterranean mechanics. Society of Petroleum Engineers Thirteenth Symposium on Oil Recovery (2002).

4. Bertsch, M. \& Bisegna, P. Blow-up of solutions of a nonlinear parabolic equation. European J. Appl. Math. 8 (1997), 89-123. Zbl 0886.35083 MR 1431415 
5. Bertsch, M. \& NitsCh, C. Traveling wave solutions of a nonlinear degenerate parabolic system from petroleum engineering. Preprint (2005).

6. Dal Passo, R., Giacomelli, L., \& GRÜN, G. A waiting time phenomena for thin film equations. Ann. Scuola Norm. Sup. Pisa 30 (2001), 437-463. Zbl 1024.35051| MR 1895718

7. Dal Passo, R., Giacomelli, L., \& GRÜN, G. Waiting time phenomena for degenerate parabolic equations-A unifying approach. Geometric Analysis and Nonlinear Partial Differential Equations, S. Hildebrandt and H. Karcher (eds.), Springer (2003), 637-648. Zbl 1028.35078 MR 2008360

8. DiBenedetto, E. Degenerate Parabolic Equations. Springer, New York (1993).

9. Ladyzhenskaya, O. A., Solonnikov, V. A., \& Ural'tseva, N. N. Linear and Quasi-Linear Equations of Parabolic Type. Transl. Math. Monographs 23, Amer. Math. Soc. (1968). Zbl 0174.15403 MR 0241821

10. Matano, $\mathrm{H}$. Nonincrease of the lap number of a solution for a one-dimensional semi-linear parabolic equation. J. Fac. Sci. Univ. Tokyo Sect. IA Math. 29 (1982), 401-441. Zbl 0496.35011 MR 0672070

11. Nitsch, C. A nonlinear parabolic system arising in damage mechanics under chemical aggression. Nonlinear Anal. 61 (2005), 695-713. Zbl pre02165053 MR 2130060

12. Nitsch, C. Some mathematical problems in nonlocal continuum damage mechanics, Ph.D. thesis (2004).

13. Simon, J. Compact sets in the space $L^{p}(0, T ; B)$. Ann. Mat. Pura Appl. 146 (1987), 65-96. Zbl 0629.46031 MR 0916688

14. StAmpacchia, G. Équations elliptiques du second ordre à coefficients discontinus. Les Presses de l’Univ. Montréal (1966). Zbl 0151.15501 MR 0251373 